\title{
Effect of COVID-19 Pandemic on the Levels of Empathy among Undergraduate Dental Students in Pakistan
}

\author{
Sundas Ghaus ${ }^{1}$ Madiha Abdul Waheed ${ }^{2}$ \\ Ali Waqar Quershi ${ }^{6}$ \\ ${ }^{1}$ Department of Dental Materials, Shifa College of Dentistry, Shifa \\ Tameer-e-Millat University, Islamabad, Pakistan \\ ${ }^{2}$ Department of Operative Dentistry, Shifa College of Dentistry, \\ Shifa Tameer-e-Millat University, Islamabad, Pakistan \\ ${ }^{3}$ Department of Biomedical Sciences, SMME, National University of \\ Science and Technology, Islamabad, Pakistan \\ ${ }^{4}$ Department of Paediatric Dentistry, Shifa College of Dentistry, \\ Shifa Tameer-e-Millat University, Islamabad, Pakistan \\ ${ }^{5}$ Department of Periodontics, Shifa College of Dentistry, Shifa \\ Tameer-e-Millat University, Islamabad, Pakistan \\ 6 Department of Prosthodontics, Shifa College of Dentistry, Shifa \\ Tameer-e-Millat University, Islamabad, Pakistan
}

Address for correspondence Shahreen Zahid Khan, BDS, MPhil, Department of Dental Materials, Shifa College of Dentistry, Shifa Tameer-e-Millat University, Islamabad 46000, Pakistan (e-mail: shahreen.scd@stmu.edu.pk).

\begin{abstract}
Keywords

- empathy

- COVID-19

- pandemic

- dentistry

- students

Objective The purpose of our study is to determine the effect of coronavirus disease 2019 (COVID-19) on the levels of empathy toward patients among undergraduate dental students in Pakistan.

Materials and Methods A cross-sectional, survey-based study was conducted at Islamic International Dental College, Islamabad, Pakistan. After taking an informed consent, a validated and pretested Toronto Empathy Questionnaire (TEQ) was distributed amongst the undergraduate dental students at two points, one before the COVID-19 existed, and the other after August 7th, 2020, when the COVID-19 cases dropped in Pakistan. Responses were indicated on a five point Likert Scale.

Statistical Analysis A one-way ANOVA (analysis of variance) was used to compare the TEQ scores between the different years of education at the dental school. Difference within the groups was analyzed using the post-hoc Tukey test. Pre- and postCOVID-19 comparison was done using the Chi-square test (significance level $p<0.05$ ). Results A total of 221 out of 260 students in the pre-COVID-19 group, while 210 out of 260 students in the post-COVID-19 group enrolled in the dentistry program participated in the study. Significant difference in the empathy levels was observed in the pre- and post-COVID-19 groups of the same population, i.e., $(p<0.05)$. The response rate was $85 \%$.

Conclusions This study showed an increase in mean empathy scores among the undergraduate dental students after COVID-19, indicating a significant improvement in their empathy levels during the time of COVID-19. It highlights the impact of the pandemic, whereby death and chaos seem to have brought about an improvement in interpersonal harmony.
\end{abstract}

DOI https://doi.org/ 10.1055/s-0040-1722091 ISSN 1305-7456. (c) 2020. European Journal of Dentistry.

This is an open access article published by Thieme under the terms of the Creative Commons Attribution-NonDerivative-NonCommercial-License, permitting copying and reproduction so long as the original work is given appropriate credit. Contents may not be used for commercial purposes, or adapted, remixed, transformed or built upon. (https://creativecommons.org/licenses/by-nc-nd/4.0/)

Thieme Medical and Scientific Publishers Pvt. Ltd., A-12, 2nd Floor, Sector 2, Noida-201301 UP, India 


\section{Introduction}

The coronavirus disease 2019 (COVID-19) outbreak in Wuhan, Hubei Province, China, in January 2020, stirred worldwide apprehension and was later declared a Public Health Emergency of International Concern by the World Health Organization (WHO) as the number of new cases and deaths from this virus continued to rise. ${ }^{1-3}$ The pandemic has challenged the health care systems around the world and has aroused different reactions and responses across the globe. ${ }^{4}$ As in all other aspects, COVID-19 has had an influence on human compassion, interpersonal harmony, and empathy levels as well.

Empathy is the capability to feel and understand another individual's feelings, experiences, problems, and viewpoint, as well as the ability to communicate understanding. ${ }^{5}$ It is a way of seeing the world from a patient's perspective. High empathy levels are known to be associated with greater contentment, reduced mental exhaustion, as well as greater psychological and occupational wellbeing. ${ }^{6,7}$ But, the question arises as to why dental health care professionals are deficient in this aspect. ${ }^{8}$ Considering the significance of "compassionate" dental care, this subject needs more emphasis. ${ }^{6}$

Several studies have been conducted to examine factors in accordance with empathy in medical and dental schools. It is alarming to note that empathy declines over years at medical and dental schools. ${ }^{9,10}$ However, what causes this decline is something to be pondered upon. Some authors believe that this is a "self-protective" response of dental practitioners that dampens compassion toward patients. Others question whether the decline in empathy is a reflection of the teaching methods adopted at dental schools. ${ }^{9}$

Authors suggest that such problems can be overcome by lifelong and continuous education of health care professionals as well as by incorporating better training programs with greater emphasis on behavioral sciences and ethics. ${ }^{11,12}$ While some authors are also of the view that empathy decline is an unavoidable psychological effect. ${ }^{13}$ The concern was captioned by one of the authors as: "How can we stop the rot?"14

Literature reports a decline in empathy levels over 4 years at a dental school. Students in year one were remarkably more empathetic than those in any of the following years. Empathy levels appeared to decline with increase in clinical exposure. .,15 $^{-15}$

Recent studies have shown behavioral and psychological changes in students amid the pandemic. ${ }^{16,17}$ Hence, it would be interesting to determine the effect of COVID-19 on the levels of empathy among undergraduate dental students.

Therefore, interestingly, although dental services were not available during the time of the pandemic, when the clinics opened and the students resumed work, a positive change in their empathy levels was observed. It gives an insight into the need to work on improving empathy levels of students in medical and dental schools. This will lead to a solution to the problem of decreasing empathy levels after increase in clinical exposure.

\section{Materials and Methods}

A cross-sectional, nonexperimental study was conducted from January 2020 to August 2020 at Islamic International Dental College, Islamabad, Pakistan. Informed consent was obtained from the students prior to the conduction of the survey. Out of the six PMDC (Pakistan Medical and Dental Council) recognized private dental colleges in Islamabad, we selected Islamic International Dental College through lottery method, in which each institution was assigned a number, and had an equal chance of being selected. ${ }^{18}$ The college was established in 2001 to contribute to the community needs of Islamabad and the surrounding areas. All undergraduate dental students enrolled in the dentistry program at Islamic International Dental College were included in the study. Participants were in either the first, second, third, or final year of the dentistry program during the 2019 to 2020 academic years. A validated and pretested Toronto Empathy Questionnaire (TEQ) was distributed to 260 undergraduate dental students (65 from each year), in the pre-COVID-19 group via face-to-face method. Data from the postCOVID-19 group was collected via a Google forms questionnaire. The study was blinded with students being unaware of the reason for re-collection of the data on the TEQ. The questionnaire was available for responses from August 7th to August 9th, 2020, at the point when the COVID-19 cases dropped in Pakistan and the lockdown situation got better. Response rate was approximately $85 \%$. Students were ensured that the information collected from them on the questionnaire will be kept confidential. Demographic information regarding "year at dental school," "gender" and "age" was included in the questionnaire. The dependent variable was empathy. Year at the dental school was the independent variable.

The TEQ consists of 16 questions that encompass a wide range of attributes associated with empathy. The TEQ exhibited good internal consistency ( $\alpha=0.87$ ) and high test-retest reliability ( 0.79 and 0.73 , respectively). The items were regarding emotional contagion $(1,4)$, emotional assessment $(2,7,10,12,15)$, empathetic response (13), emotion comprehension (8), sympathetic physiological arousal $(3,6,9$, $11)$, and altruism $(5,14,16)$. Empathy was assessed with a five point Likert scale ranging from "never" to "always". Item responses were scored according to the following scale for positively worded items $1,3,5,6,8,9,13,16$, i.e., never $=0$; rarely $=1$; sometimes $=2$; often $=3$; always $=4$. The following negatively worded items were reverse scored: $2,4,7$, $10,11,12,14$, and 15 , i.e., 0 = always, 1 = often, 2 = sometimes, 3 = rarely, and $4=$ never. Scores were summed to derive total for the TEQ. Maximum possible score was 64, while the least possible score was 0 . Higher scores indicated higher levels of empathy. ${ }^{19}$ The data was then entered in SPSS version 23. A one-way ANOVA (analysis of variance) was used to compare the TEQ scores between the different years of education at the dental school. Difference within the groups was analyzed using the post-hoc Tukey test. Pre- and post-COVID-19 comparison was done using the Chi-square test. Statistical significance was set at $p<0.05$. Statistical 
analysis was performed using SPSS for windows software (version 23.0).

\section{Results}

A total of 221 out of 260 students in the pre-COVID-19 group, while 210 out of 260 students in the post-COVID-19 group enrolled in the undergraduate dentistry program at Islamic International Dental College, Islamabad, Pakistan, participated in the study. Response rate was approximately $85 \%$. An accepted Cronbach $\alpha$ of 0.7 was calculated of the complete questionnaire. The distribution of students in the preCOVID-19 group was as follows: 58 (26\%) from first year, 57 (25\%) from second year, 57 (25\%) from third year, and 49 (22\%) from the final year of the dentistry program (-Fig. 1A). Post-COVID-19 group distribution was as follows: 42 (20\%) from first year, 59 (29\%) from second year, 68 (32\%) from third year, and 41 (19\%) from the final year (-Fig. 2A). Male to female ratio was 1:3 (-Figs. 1B and 2B). Distributions of the participants' gender and educational level are shown in - Table 1. Age-wise distribution of the students at the dental school is illustrated in -Figs. $\mathbf{1 C}$ and $\mathbf{2 C}$.

In the pre-COVID-19 group, the mean values of the first year and the second year showed almost similar empathy at 2.90 and 2.97, respectively, which were still higher than that of the third year (2.88), while significantly higher than that of final year (2.48) (-Fig. 1D).On the contrary, the post-COVID-19 group showed the following trend: mean empathy scores of final year was 3.07, which was close to that of the first year (3.05), while significantly higher than that of the second and the third year (2.98) ( - Fig. 2D). These distributions are illustrated in - Table 2.

A one-way ANOVA was used to compare the TEQ scores between the different years of education at the dental school for both the pre- and the post-COIVD groups. Statistical significance was set at $p<0.05$. Significant difference in the empathy levels between the groups was observed in the pre-COVID-19 group (0.000), while the post-COVID-19 group of the same population showed no significant difference between the different years $(0.171)$ at $p<0.05$. ( Table 3 )
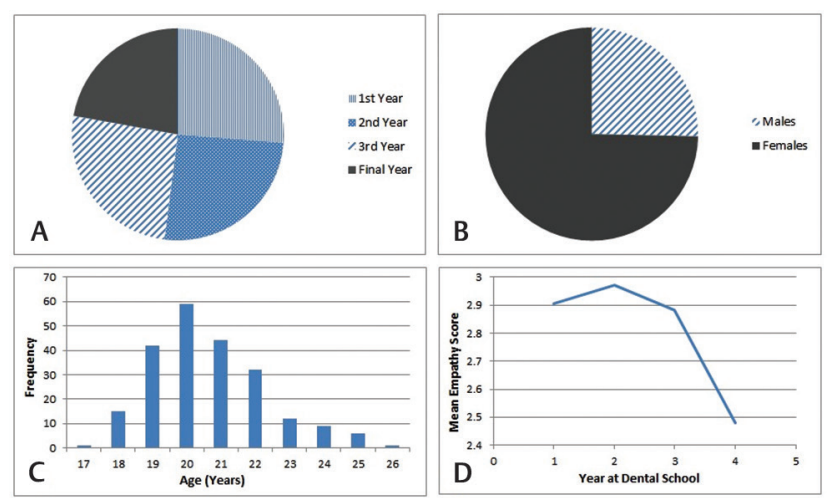

Fig. 1 Pre-COVID-19 group (A) year at dental school, (B) gender-wise distribution of students, (C) age-wise distribution of students, (D) mean empathy scores of students from each year. COVID-19, coronavirus disease 2019.
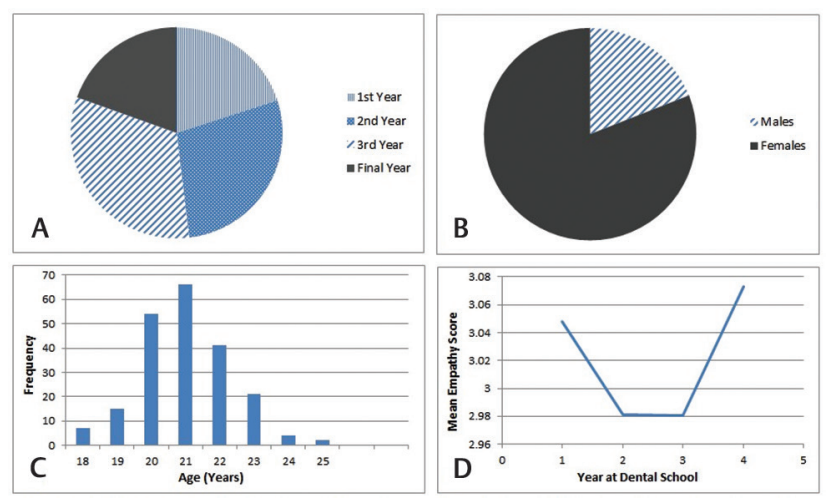

Fig. 2 Post-COVID-19 group (A) year at dental school, (B) gender-wise distribution of students, (C) age-wise distribution of students, (D) mean empathy scores of students from each year. COVID-19, coronavirus disease 2019.

In the pre-COVID-19 group, although there was no significant difference between the first and second year, second and third year, after post-hoc analysis using Tukey, significant difference was observed between the first 3 years and the final year at $p<0.05$. While in the postCOVID-19 group, post-hoc analysis using Tukey showed no significant difference between the different years at the dental school.

To sum up, there was a significant difference between the results of the pre- and the post-COVID-19 data which showed a significant improvement in the empathy levels of the students during the time of COVID-19 (- Table 3). Hence it is stated that the empathy levels changed during the time of the pandemic.

\section{Discussion}

Literature review of the studies conducted prior to the outbreak of the pandemic shows trends similar to those observed in our study.

A cross-sectional study performed at CMH Lahore Medical College and Institute of Dentistry, Pakistan, showed a decline in mean empathy scores among dentistry students as the years of study progressed. ${ }^{5}$ A similar study at University of Washington School of Dentistry, United States, concluded that empathy levels declined as clinical exposure increased. Levels were highest during the first year and showed a decline during the following years. ${ }^{10}$ In another study, a statistically significant drop in empathy levels in medical students from the beginning to the end of their third year at medical school was observed. ${ }^{15} \mathrm{~A}$ systematic review of literature showed similar results. ${ }^{10}$

Another study conducted at an established dental college and hospital in Islamabad, Pakistan, showed similar trends. Hospitality and empathy levels were seen to decline and third year students were found to be least empathetic. They conducted a survey toward the end of each academic year. ${ }^{20}$

Furthermore, a cross-sectional study performed at Department of Family Medicine, Ondokuz Mayas University, Samsun, Turkey, gave the same conclusion. ${ }^{21}$ 
Table 1 Distribution of participants' educational level and gender at IIDC $(\mathrm{N}=260)$

\begin{tabular}{|l|l|l|l|}
\hline \multicolumn{5}{|c|}{ Pre-COVID-19 group } & \multicolumn{2}{c|}{ Post-COVID-19 group } \\
\hline Variables & $N(\%)$ & Variables & $N)$ \\
\hline First year & $58(26 \%)$ & First year & $42(20 \%)$ \\
\hline Second year & $57(25 \%)$ & Second year & $59(29 \%)$ \\
\hline Third year & $57(25 \%)$ & Third year & $68(32 \%)$ \\
\hline Final year & $49(22 \%)$ & Final year & $41(19 \%)$ \\
\hline Total & 221 & Total & 210 \\
\hline Gender & & & \\
\hline Males & $56(25 \%)$ & Males & $40(19 \%)$ \\
\hline Females & $165(75 \%)$ & Females & $170(81 \%)$ \\
\hline
\end{tabular}

Abbreviation: COVID-19, coronavirus disease 2019.

Table 2 Year-wise distribution of mean empathy scores and standard deviations

\begin{tabular}{|l|l|l|l|}
\hline \multicolumn{2}{|c|}{ Pre-COVID-19 group } & \multicolumn{2}{c|}{ Post-COVID-19 group } \\
\hline Year at dental school & Mean empathy score (SD) & Year at dental school & Mean empathy score (SD) \\
\hline First year & $2.9063(1.17270)$ & First year & $3.0476(1.03477)$ \\
\hline Second year & $2.9726(1.13826)$ & Second year & $2.9809(1.05202)$ \\
\hline Third year & $2.8838(1.09368)$ & Third year & $2.9807(1.04393)$ \\
\hline Final year & $2.4783(1.34175)$ & Final year & $3.0732(0.94787)$ \\
\hline
\end{tabular}

Abbreviation: COVID-19, coronavirus disease 2019.

Table 3 One-way ANOVA and Chi-square analysis

\begin{tabular}{|l|l|l|l|l|}
\hline \multicolumn{3}{|c|}{ One-way ANOVA } \\
\hline \multicolumn{3}{|c|}{ Pre-COVID-19 group } & \multicolumn{3}{c|}{ Post-COVID-19 group } \\
\hline Between groups & Sig. & & Sig. \\
\hline \multicolumn{2}{|c|}{ Pre vs. post-COVID-19 group comparison } & 0.171 \\
\hline & 0.000 & Value & $\mathrm{d} f$ & \multicolumn{1}{c|}{ Asymptotic significance (two-sided) } \\
\hline Pearson Chi-square & $49.824^{\text {a }}$ & 16 & 0.000 \\
\hline
\end{tabular}

Abbreviations: ANOVA, analysis of variance; COVID-19, coronavirus disease 2019

${ }^{\mathrm{a}}$ Cells $(4.0 \%)$ have expected count less than 5 . The minimum expected count is 4.70 .

Research generally shows that the third year is typical when students often deal with emotionally challenging and difficult situations while rotating through the various clinical departments. Their empathy declines as a protective defense mechanism. ${ }^{21}$ Another study performed in two private dental institutions in India showed a similar decline at the time of the clinical phase of training. ${ }^{15}$

A cross-sectional descriptive study on Indian dental students showed much lower mean empathy levels. Cultural and ethnical differences were reported to be the reason behind the disparity.22

On the contrary, a cross-sectional study at two universities in Australia showed that empathy scores increased from the first year to the second year of study, but did not significantly increase/decrease after this. Statistically significant differences were seen between certain age groups. ${ }^{23}$

Another cross-sectional study on empathy conducted at King Abdulaziz University, Saudi Arabia, found that dental students were empathetic although their mean empathy score was not as high as expected. The fifth and sixth year students had higher scores than the third and fourth year students although the differences were not statistically significant. ${ }^{24}$

Furthermore, a study on Polish dental students showed an increase in empathy from the first and second to fourth years, where it reached its maximum. Levels decreased during the fifth year with students having to meet a set target to finish the course. ${ }^{25}$ Bachelor of dental surgery in Pakistan is a 4-year program.

Surprisingly however, the trends took a turn as the COVID-19 pandemic took its toll across the globe.

The survey distribution period of the postCOVID-19 group was selected to match the time when the COVID-19 cases dropped in Pakistan and the national lockdown situation got better. The subsequent prevalence of higher mean empathy scores in the post-COVID-19 group 
will have a positive impact on dental as well as on generalized human care.

The focus of this study before the COVID-19 outbreak was to observe the trends in empathy seen during the 4 years of the undergraduate program at the dental school, but, after the sudden change in events due to the outbreak, when the data was re-collected from the same population, surprising results were obtained. Therefore, the present study not only calculated the change in empathy levels between the first and the final year which was evident previously, but it also showed a marked and significant improvement in the empathy levels of the students after facing the pandemic. This suggests that when the students were staying at home, attending online sessions, ${ }^{26}$ and were off-campus and no longer exposed to the patients, the existing COVID-19 environment did have a positive impact on their mean empathy scores.

Recent literature reports that the spirit of empathy among health care providers was linked with better outcomes particularly at the peak time of the pandemic when the health care systems of several countries were on the verge of total collapse. ${ }^{27} \mathrm{~A}$ recent study involving 23 different countries across the globe showed a significant and positive attitude of dental health professionals toward disinfection against COVID-19, although the dentists showed insufficient knowledge about the essential aspects of disinfection protocols. ${ }^{28,29}$ The pandemic also encouraged research into understanding people's emotional reactions when experiencing life-threatening events, thereby confirming the benefits of restoring interpersonal connections and effective health communication during health emergencies like the COVID-19.30,31

However, amid the COVID-19 crisis, anxiety has been exacerbated in the population globally, leading to mental health disorders in individuals. ${ }^{16,32}$ Mental health disorders negatively impact learning and academic progress. ${ }^{33}$ Evidence suggests that individuals may experience symptoms of psychosis, anxiety, trauma, suicidal thoughts, and panic attacks. ${ }^{34}$ Recent studies report the same. ${ }^{35-39}$ In health care workers who treated patients with COVID-19, social support did have a positive impact on sleep quality and efficacy, thereby causing a reduction in anxiety and stress. ${ }^{40}$

Further studies need be conducted to observe if the results from the present study can be generalized to other medical and dental institutions of the country as the pandemic is a national as well as an international challenge. But, collection of data from the same population retrospectively is unlikely and hence it is very rare to find studies with such comparisons, particularly on the basis of empathy.

Thus, the present study highlights the significant impact of COVID-19 on undergraduate dental students at Islamic International Dental College, Islamabad. Interestingly, being exposed to a similar course online, the same population of students showed a reversal in the trend-whereby the final year students re-gained the empathy levels lost over the first 3 years at the dental school. Additionally, a generalized improvement in the empathy levels was observed. Therefore, results from the present study have opened a new era that calls for insight by the academic faculty and the governance into the on-campus academic environment to address the decreasing empathy levels over the years.

\section{Conclusion}

To conclude, dental students from all the 4 years of the undergraduate program at the dental school showed a significant improvement in the empathy levels following the pandemic. The results highlight the impact of the pandemic on human life, whereby death and chaos seem to have brought about a change in attitudes and behavior, and an improvement in interpersonal harmony. However, the longterm psychosocial impact of this virus outbreak on dental students remains unknown. Therefore, emphasis needs to be placed on incorporating programs on behavioral sciences and ethics, and on on-campus mentoring during the undergraduate years, which can bring about an improvement in overall patient care.

\section{Conflict of Interest}

None declared.

\section{References}

1 Wang C, Horby PW, Hayden FG, Gao GF. A novel coronavirus outbreak of global health concern. Lancet 2020;395(10223):470-473

2 Huang C, Wang Y, Li X, et al. Clinical features of patients infected with 2019 novel coronavirus in Wuhan, China. Lancet 2020;395(10223):497-506

3 Meng L, Hua F, Bian Z. Coronavirus disease 2019 (COVID-19): emerging and future challenges for dental and oral medicine. J Dent Res 2020;99(5):481-487

4 Coulthard P. Dentistry and coronavirus (COVID-19)-moral decision-making. Br Dent J 2020;228(7):503-505

5 Kamran R, Bari A, Ijaz F, et al. Levels of empathy among dental students at $\mathrm{CMH}$ Lahore Medical College and Institute of Dentistry (NUMS) Lahore Pakistan. Int J Med Res Heal Sci 2019;8(8):14-18

6 Jeffrey D. Empathy, sympathy and compassion in healthcare: is there a problem? Is there a difference? Does it matter? J R Soc Med 2016;109(12):446-452

7 Lamiani G, Dordoni P, Vegni E, Barajon I. Caring for critically ill patients: clinicians' empathy promotes job satisfaction and does not predict moral distress. Front Psychol 2020;10:2902

8 Haslam D. More than kindness. J Compassionate Health Care 2015;2(1):6

9 Huang L, Thai J, Zhong Y, Peng H, Koran J, Zhao XD. The positive association between empathy and self-esteem in Chinese medical students: a multi-institutional study. Front Psychol 2019;10:1921 doi:10.3389/fpsyg.2019.01921

10 Narang R, Mittal L, Saha S, Aggarwal VP, Sood P, Mehra S. Empathy among dental students: a systematic review of literature. J Indian Soc Pedod Prev Dent 2019;37(4):316-326

11 Souror YR, Aljehani DK, Alshaikh MH. Empathy of dental students towards children after behaviour guidance lectures and clinical experience. Eur J Dent Educ 2020;24(3):458-464

12 Mac Giolla Phadraig C, Griffiths C, McCallion P, McCarron M, Wride MA, Nunn JH. How dentists learn behaviour support skills for adults with intellectual developmental disorders: a qualitative analysis. Eur J Dent Educ 2020;24(3):535-541

13 Moudatsou M, Stavropoulou A, Philalithis A, Koukouli S. The role of empathy in health and social care professionals. Healthcare (Basel) 2020;8(1):26 
14 Hudson JN, Lethbridge A, Vella S, Caputi P. Decline in medical students' attitudes to interprofessional learning and patient centredness. Med Educ 2016;50(5):550-559

15 Aggarwal VP, Garg R, Goyal N, et al. Exploring the missing link-empathy among dental students: an institutional cross-sectional survey. Dent Res J (Isfahan) 2016;13(5):419-423

16 Salari N, Hosseinian-Far A, Jalali R, et al. Prevalence of stress, anxiety, depression among the general population during the COVID-19 pandemic: a systematic review and meta-analysis. Global Health 2020;16(1):57

17 Taylor MR, Agho KE, Stevens GJ, Raphael B. Factors influencing psychological distress during a disease epidemic: data from Australia's first outbreak of equine influenza. BMC Public Health 2008;8:347

18 Yilmaz HG, Kurtulmus-Yilmaz S, Cengiz E, Bayindir H, Aykac Y. Clinical evaluation of Er, Cr:YSGG and GaAlAs laser therapy for treating dentine hypersensitivity: a randomized controlled clinical trial. J Dent 2011;39(3):249-254

19 Alhadidi MM, Abdalrahim MS, Al-Hussami M. Nurses' caring and empathy in Jordanian psychiatric hospitals: A national survey. Int J Ment Health Nurs 2016;25(4):337-345

20 Javed MQ. The evaluation of empathy level of undergraduate dental students in Pakistan: A cross-sectional study. J Ayub Med Coll Abbottabad 2019;31(3):402-406

21 Igde FA, Sahin MK. Changes in empathy during medical education: An example from Turkey. Pak J Med Sci 2017;33(5):1177-1181

22 Prabhu S, Prasanth SS, Kishore S, Kumar VS. Standing in patients' shoes-survey on empathy among dental students in India. J Educ Ethics Dent 2014;4(2):69-73

23 Williams B, Brown T, McKenna L, et al. Empathy levels among health professional students: a cross-sectional study at two universities in Australia. Adv Med Educ Pract 2014;5:107-113

24 Naguib G, Sindi A, Attar M, Alshouibi E, Hamed M. A cross-sectional study of empathy among dental students at King Abdulaziz University. J Dent Educ 2019;83:e1-e5

25 Mocny-Pachońska K, Łanowy P, Trzcionka A, Skaba D, Tanasiewicz M. Gender related changes of empathy level among Polish dental students over the course of training. Medicine (Baltimore) 2020;99(1):e18470

26 Sarwar H, Akhtar H, Naeem MM, et al. Self-reported effectiveness of e-learning classes during COVID-19 pandemic: A nation-wide survey of Pakistani undergraduate dentistry students. Eur J Dent 2020;14(suppl S1):S34-S43 doi:10.1055/ s-0040-1717000

27 Barello S, Palamenghi L, Graffigna G. Empathic communication as a "Risky strength" for health during the COVID-19 pandemic:
The case of frontline Italian healthcare workers. Patient Educ Couns 2020;103(10):2200-2202

28 Sarfaraz S, Shabbir J, Mudasser MA, et al. Knowledge and attitude of dental practitioners related to disinfection during the COVID-19 pandemic. Healthcare (Basel) 2020;8(3):232

29 Azim AA, Shabbir J, Khurshid Z, Zafar MS, Ghabbani HM, Dummer PMH. Clinical endodontic management during the COVID-19 pandemic: a literature review and clinical recommendations. Int Endod J 2020;53(11):1461-1471 doi:10.1111/ iej.13406

30 Barello S, Graffigna G. Caring for health professionals in the COVID-19 pandemic emergency: toward an "epidemic of empathy" in healthcare. Front Psychol 2020;11:1431

31 Finset A, Bosworth H, Butow P, et al. Effective health communication-a key factor in fighting the COVID-19 pandemic. Patient Educ Couns 2020;103(5):873-876

32 Ahmed MA, Jouhar R, Ahmed N, et al. Fear and practice modifications among dentists to combat novel coronavirus disease (COVID-19) outbreak. Int J Environ Res Public Health 2020;17(8):E2821

33 Araújo FJO, de Lima LSA, Cidade PIM, Nobre CB, Neto MLR. Impact of SARS-CoV-2 and its reverberation in global higher education and mental health. Psychiatry Res 2020;288:112977

34 World Health Organization. Available at: https://apps.who. int/iris/bitstream/handle/10665/331490/WHO-2019-nCoVMentalHealth-2020.1-eng.pdf. Accessed September 19, 2020

35 Ahmed MZ, Ahmed O, Aibao Z, Hanbin S, Siyu L, Ahmad A. Epidemic of COVID-19 in China and associated psychological problems. Asian J Psychiatr 2020;51:102092

36 Cao W, Fang Z, Hou G, et al. The psychological impact of the COVID-19 epidemic on college students in China. Psychiatry Res 2020;287:112934

37 Wang Y, Di Y, Ye J, Wei W. Study on the public psychological states and its related factors during the outbreak of coronavirus disease 2019 (COVID-19) in some regions of China [published online ahead of print, 2020 Mar 30]. Psychol Health Med 2020;1-10 doi:10.1080/13548506.2020.1746817

38 Huang Y, Zhao N. Generalized anxiety disorder, depressive symptoms and sleep quality during COVID-19 outbreak in China: a web-based cross-sectional survey. Psychiatry Res 2020;288:112954

39 Rajkumar RP. COVID-19 and mental health: a review of the existing literature. Asian J Psychiatr 2020;52:102066

40 Xiao H, Zhang Y, Kong D, Li S, Yang N. The effects of social support on sleep quality of medical staff treating patients with coronavirus disease 2019(COVID-19) in January and February 2020 in China. Med Sci Monit 2020;26:e923549-e1 